\title{
Problemas teóricos, metodológicos e instrumentales para el estudio de la calidad de vida en personas con VI H en Angola
}

\section{Theoretical, methodological and instrumental problems for the study of quality of life in HIV persons in Angola}

\author{
MsC. Lucas Antonio Nhamba,' DraC. Edelsys Hernández Meléndrez,' \\ DrC. Héctor Demetrio Bayarre Vea,' DrC. Maria Gamba Janota" \\ ' Escuela Nacional de Salud Pública. La Habana, Cuba. \\ "Universidad "Agostinho Neto". Angola.
}

\section{RESUMEN}

El estudio de la calidad de vida es uno de los problemas que merecen especial atención en la actualidad en las ciencias de la salud ante el desarrollo científico, tecnológico y la transición epidemiológica. Su investigación requiere de un abordaje multidisciplinario e intersectorial. EI VIH es un problema que afecta la calidad de vida de la población angoleña. Los problemas teóricos, metodológicos e instrumentales de la investigación sobre la calidad de vida en personas con VIH, así como sus principales tendencias, se discuten mediante un enfoque deductivo. Uno de los problemas de este constructo es la falta de consenso entorno a su definición conceptual, desde diferentes disciplinas, base de las falencias metodológicas que han dado paso a la proliferación de instrumentos genéricos y específicos para el estudio de la calidad de vida relacionada con la salud y la enfermedad. Así, se arriba al estado actual y perspectiva de su estudio en la población angolana. Se proponen alternativas para una investigación multidisciplinaria sobre la calidad de vida en personas con $\mathrm{VIH}$, punto de partida para la elaboración de estrategias para su mejoramiento.

Palabras clave: Calidad de vida, calidad de vida relacionada con la salud, personas con VIH. 


\section{ABSTRACT}

The study of the quality of life is one of the problems deserving special attention in the health sciences at present due to the scientific and technological development and to the epidemiological transition. The research on this topic requires multidisciplinary and intersectoral approach. HIV is a problem having an impact on the quality of life of the Angolan population. This article discussed with deductive approach the theoretical, methodological and instrumental problems in research as well as the main tendencies in the research of the quality of life in HIV persons. One of the difficulties in this construct is the lack of consensus around its conceptual definition by different disciplines, which leads to the proliferation of generic and specific instruments to study the quality of life associated to health and disease. This is the present state and perspective of the quality of life study in the Angolan population. Some alternatives are submitted in order to conduct a multidisciplinary research study on the quality of life of HIV persons, the starting point to draw adequate strategies for its improvement.

Key words: Quality of life, health-related quality of life, HIV people.

\section{NTRODUCCI ÓN}

El interés por la calidad de vida no es reciente y ha sido expresado por diferentes formas de actuación, en diversos momentos históricos y culturales de los pueblos. ${ }^{1-}$ ${ }^{3}$ Es para Hernández Meléndrez un concepto que presenta dificultades para su operacionalización y ha sido motivo de debates acerca de su naturaleza objetiva y subjetiva, de la delimitación de enfoques afines, de su estructura y de los métodos e instrumentos para su estudio. ${ }^{1}$

A pesar de la existencia de falta de consenso en cuanto al concepto, las dimensiones y sobre los diferentes instrumentos para evaluar la calidad de vida relacionada con la salud, la importancia de su estudio es reconocida por la mayoría de los investigadores por aportar informaciones valiosas que complementan los registros de síntomas o pruebas funcionales tradicionales. ${ }^{4}$

Se muestra también como una herramienta importante en la evaluación de estrategias de tratamiento y gestión de los servicios de salud, ${ }^{5}$ y como guía para el establecimiento de políticas para los cuidados en salud. Su aplicación en las enfermedades crónicas como el VIH, es de amplio reconocimiento. ${ }^{6}$

El concepto de calidad de vida relacionado con la salud, se refiere a una categoría que permite de forma cualitativa ponderar las condiciones en que el individuo, un determinado grupo, una comunidad o la sociedad en su conjunto, se desempeña en la vida frente a los procesos que puedan afectar su salud y la terapéutica empleada en su restablecimiento en el dominio físico, psíquico, y social.

EI VIH/sida en Angola constituye un grave problema de salud por su alta morbilidad y mortalidad, a lo que se adicionan los problemas sociales que esta epidemia ha generado, entre los que se destacan mitos y falsas creencias entorno a la 
enfermedad que afectan la percepción de la calidad de vida en este segmento de la población.

Lo planteado con anterioridad pone de manifiesto la necesidad del estudio de la calidad de vida en personas con VIH. Sin embargo, a los problemas señalados para su estudio, se le adiciona la situación del contexto y la carencia de procedimientos e instrumentos autóctonos y específicos, lo que limita su abordaje e impone la elaboración de alternativas encaminadas a mitigar sus efectos negativos.

El propósito de este trabajo es el análisis de los problemas teóricos, metodológicos e instrumentales del estudio de la calidad de vida en personas con VIH en Angola, a partir de las limitaciones generales señaladas para la investigación del constructo. Ello deviene un reto para el diseño de estrategias encaminadas a su mejoramiento.

\section{EL PROBLEMA}

Una de las mayores controversias entre los investigadores según Hernández, surge por el hecho de que no todos se refieren a los mismos aspectos. Cuando se revisa la literatura en Internet, puede notarse que existen infinidad de artículos que se refieren a la categoría calidad de vida, pero en realidad se refieren a otras categorías con ella relacionada como: modo de vida, estilo de vida, género de vida y nivel de vida, que aunque estrechamente vinculadas tienen su diferenciación. ${ }^{1}$

En la actualidad, con el creciente desarrollo tecnológico de la medicina y ciencias afines, la gran preocupación con el concepto de calidad de vida se refiere a un movimiento dentro de las ciencias humanas y biológicas en el sentido de valorar parámetros más amplios, que sobrepasan el control de síntomas, la disminución de la mortalidad o el aumento de la expectativa de vida., ${ }^{7,8}$

La expresión antedicha invade los medios de comunicación, por lo que el constructo de calidad de vida toma diversas connotaciones, ante todo de carácter personalista y de valor, por lo que asume una perspectiva sociocultural, también acompañada de pensamiento ecologista. ${ }^{9}$

A mediados de los años 70 y comienzo de los 80 con el desarrollo y perfeccionamiento de los indicadores sociales, se da un proceso de diferenciación entre estos y la calidad de vida. La expresión comienza a definirse como concepto integrador que comprende todas las áreas de la vida, por lo que adquiere un carácter multidimensional y hace referencia tanto a condiciones objetivas, como subjetivas.

En la actualidad, se tienen referencias históricas de su utilización mediante conceptos similares como los de salud, bienestar y felicidad. ${ }^{10}$ Hoy el concepto está popularizado y se ha tornado objeto de interés de diferentes disciplinas. ${ }^{11}$

La calidad de vida es así entendida como un concepto profundamente condicionado por la cultura, y con una estricta dependencia del conjunto de valores de los individuos y de los grupos sociales. De esta forma, resulta difícil acotar, pues se constituye socialmente como la representación que un colectivo puede tener sobre su propia vida. ${ }^{11}$

No obstante las discrepancias que el concepto presenta para su abordaje, González $\mathrm{U}^{2}$ reconoce que la calidad de vida posee un carácter integrador que permite 
armonizar la información que se obtiene de otras disciplinas e investigaciones con otros objetivos, metodologías y técnicas diferentes. Se refiere a la importancia del estudio de la calidad de vida para intervenir en la enfermedad, la salud y el bienestar, la considera una muestra de progreso de las ciencias y las humanidades $y$, por lo tanto, debe ser aprovechada trabajando en su fundamentación teórica y metodológica, en sus conceptos y terminologías, en la construcción de instrumentos y en la búsqueda de su aplicación y eficacia. ${ }^{2}$

De acuerdo con Bayarre, en la actualidad estamos frente a un anhelo de la humanidad, que es la prolongación de la vida, que ha resultado de varias conquistas en el campo social y científico. ${ }^{12}$

El desarrollo de las ciencias médicas permitió un incremento de la esperanza de vida y con ello de las enfermedades crónicas. Ello enfatiza la necesidad de dar especial atención a un nuevo término: calidad de vida relacionada con la salud (CVRS). En la actualidad numerosos estudios emplean este término para referirse a la percepción que tiene el paciente de los efectos de una enfermedad determinada - de la aplicación de cierto tratamiento en diversos ámbitos de su vida, especialmente de las consecuencias que provoca sobre su bienestar físico, emocional y social. ${ }^{13}$

No obstante su amplia utilización como una medida del estado de salud de las personas, Urzúa refiere que en el campo de la salud, la CVRS ha sido el concepto más utilizado y de forma indistinta al de calidad de vida general. Considera que a pesar de la falta de consenso en la definición de este término, la información generada de su estudio se constituye en un gran insumo para la formulación de objetivos, guías y políticas para los cuidados en salud, y también su importancia es reconocida en la descripción del impacto de la enfermedad en la vida de los pacientes, así como en la evaluación de la efectividad de los tratamientos. ${ }^{6}$

Dando realce a la importancia del estudio sobre la evaluación de la CVRS, en cuanto una variable resultante, Miguel $L$ y otros, refieren que este término tiene una presencia cada vez mayor en la investigación clínica por aportar informaciones valiosas que complementan los registros de síntomas o pruebas funcionales tradicionales. ${ }^{4}$ Su mayor valor radica en que se trata de una medición que se hace sobre la salud desde la perspectiva del propio paciente, ${ }^{6}$ considera además, que hay muchas cuestiones pendientes, relacionadas con la necesidad de un sólido modelo conceptual y una mejor especificación de sus dimensiones, lo que ayudaría a interpretar mejor el sentido y significado de los resultados obtenidos en las distintas investigaciones. ${ }^{4-6}$

Según Canini, la mensuración de la calidad de vida, también se constituye en una herramienta importante, pues permite la evaluación de estrategias de tratamiento y costo/beneficio, y direccionar la distribución de recursos e implementación de programas de salud que pueden privilegiar los aspectos físicos de los pacientes y los relacionados con las dimensiones psíquicas y sociales que permite a los equipos de salud planificar la atención integral. ${ }^{5}$

Entre los grandes logros de los últimos decenios en la rama de la medicina, con enormes victorias en las enfermedades infecciosas y en la prolongación de la vida, se encuentran la revolución terapéutica, la revolución biológica en el ámbito de la genética en la lucha contra enfermedades hereditarias y el cáncer. La medicina en la actualidad, no satisfecha con los éxitos que se pueden calcular en términos cuantitativos, se sitúa en el campo de la investigación de la calidad de vida, como una forma de enfatizar mucho más en la humanización de los servicios asistenciales. ${ }^{9,14}$ 
La indefinición conceptual y metodológica en torno a la calidad de vida ha influido en el aspecto tecnológico, los autores no se ponen de acuerdo en cuanto a las formas de evaluarla, y esta diversidad de instrumentos, generados por unos y otros, a su vez, ha redundado negativamente en la clarificación del constructo. ${ }^{15}$

De esta forma, para la medición de los nuevos parámetros, a la luz de un nuevo abordaje en los cuidados de los pacientes, emergieron varias escalas para medir la calidad de vida pero, muchas de ellos pecan por falta de idoneidad. ${ }^{16}$

A los problemas conceptuales que directamente implican las escalas e instrumentos, se suman las controversias sobre cuáles son los elementos objetivos y subjetivos en la calidad de vida, surgiendo de ahí la cuestión sobre a quién compete evaluar la calidad de vida, y la determinación de su jerarquía, su magnitud y su importancia. ${ }^{14-16}$

Sobre esta cuestión, los investigadores sobre el tema son unánimes en reconocer que la evaluación de los parámetros objetivos, físicos y de salud evidentemente le competen al médico o profesional de salud, pero la valoración de los factores psicológicos, tales como la expectativa sobre los cuidados que espera en relación con los servicios, percepción de sí, su bienestar y seguridad, es de total competencia del paciente. ${ }^{9,15}$

A pesar de las dificultades planteadas por muchos estudiosos sobre el tema, que Urzúa llamó "carencias", pero que reconoce la valiosa utilidad de la calidad de vida en las enfermedades crónicas ya que las mediciones clínicas/fisiológicas tradicionales proveen informaciones para el clínico, con interés limitado para el paciente, por lo que ofrece de esta forma un correlato pobre entre la capacidad funcional y el bienestar, área de mayor interés para el paciente.

Estas dificultades que se han analizado están muchas veces relacionadas con la súper especialización, muchas veces no se buscan los elementos generales, sino que cada rama quiere desarrollar su propia tecnología, por ejemplo, se aspira a establecer tantas escalas de calidad de vida como especialidades existen y no los elementos comunes en cuanto a sus indicadores. Es así como han surgido escalas para evaluar la calidad de vida en pacientes con cáncer, con asma, con cardiopatías, entre otras enfermedades.

Hace casi tres décadas la humanidad confronta una de las mayores epidemias, la producida por el virus de inmunodeficiencia humana, que produce efectos heterogéneos en diferentes continentes y países. Las posibles soluciones a la pandemia siguen desafiando todo el desarrollo tecnológico y científico alcanzado por la humanidad, de ahí que se ha transformado en uno de los mayores problemas de salud en el mundo.

El VIH/sida está teniendo un efecto devastador en los más diversos dominios de la vida de las poblaciones. Por ejemplo, ha reducido hasta en 20 años la expectativa de vida de las personas, por lo que tiene un fuerte impacto sobre la economía al dejar más de 12 millones de huérfanos, lo que ha su vez, ha generando un gran impacto en la distribución de las personas por grupos de edades. Lo anterior implica grandes riesgos en la transferencia de conocimientos y valores de una generación a otra. ${ }^{17}$

Hoy, las personas sufren el peso doble de la enfermedad, caracterizada por las pérdidas reales, por muerte de miembros de la familia y también por la "muerte social" causada por la discriminación de personas infectadas y familias afectadas. Esto lleva a la necesidad de que los equipos de salud sean mejores desde el punto 
de vista tecnológico y se sensibilicen ante el sufrimiento que provoca la noticia de una enfermedad que está estigmatizada como un padecimiento que irremediablemente llevará al fin de las vidas de las personas, a dejar a sus padres sin protección y sus hijos sin amparo.

Esta situación tiene reforzado el impacto nefasto de la enfermedad, por lo que exige la movilización de personas, familias y organizaciones nacionales e internacionales en la búsqueda de estrategias para su enfrentamiento y la asociación a las incansables pesquisas en búsqueda de tratamiento y de la mejor calidad de vida de las personas viviendo con VIH o ya enfermas con el sida.

Naciones Unidas, por medio de la ONUSIDA, estima que 33,3 millones de personas viven con el virus de la inmunodeficiencia. Reconoce que en los días actuales, la epidemia del sida ha comenzado a desacelerarse con una reducción en el número de contagios y en las muertes provocadas por la enfermedad. ${ }^{17}$

Los fracasos, en la solución del problema, se deben a la falta de correspondencia entre las estrategias contra el sida y las necesidades nacionales identificadas. Se refiere a poca inversión en programas de prevención dirigidos a grupos con mayor riesgo (los usuarios de droga inyectables, hombres que tienen relaciones sexuales con hombres, los profesionales del sexo, los reclusos y trabajadores migratorios), brechas que verifican una escasez habitual de programas específicamente diseñados para personas que viven con el $\mathrm{VIH} .{ }^{17}$

En Angola la epidemia de $\mathrm{VIH} /$ sida ha continuado su ritmo ascendente, probablemente a partir del perfeccionamiento de los medios diagnósticos, del incremento de la red de servicios de salud destinados a la atención de este problema y a los avances en la conducta terapéutica. Sin embargo, ello no ha modificado los mitos y creencias erróneas generadas desde su aparición, con sus efectos negativos sobre los estados emocionales, sus mecanismos de afrontamiento que afectan el nivel de relaciones familiares y sociolaborales de quienes la padecen, con la consecuente repercusión sobre su salud y su calidad de vida. ${ }^{18,19}$

Lo planteado con anterioridad, constituye un argumento para el estudio del constructo. A pesar de ello, su abordaje transita por las mismas dificultades señaladas, incrementadas por la carencia de estudios anteriores en el contexto angoleño y la falta de instrumentos específicos para el problema de referencia. Ello constituye un reto para este grupo de investigadores que, pretende encontrar alternativas metodológicas e instrumentales que hagan factible su estudio.

\section{CONSI DERACI ONES FI NALES}

Se encontraron tres grandes grupos de problemas al abordar el desarrollo de los estudios de la calidad de vida en personas con VIH en Angola:

1. El primero es el referido a su indistinción conceptual, determinado en gran parte por su naturaleza compleja. Como resulta con muchos otros constructos, ha resultado más fácil medirla que definirla. Se trabaja en una definición conceptual que abarque todos los atributos de la calidad de vida relacionada con la salud en personas con VIH. 
2. Asociado a lo anterior, los problemas relacionados con la naturaleza de la calidad de vida, de sus componentes subjetivos y objetivos. No hay duda de que la calidad de vida es el resultado de la compleja interacción entre factores objetivos y subjetivos, los primeros constituyen las condiciones externas: económicas, sociopolíticas, culturales y ambientales que facilitan o entorpecen el pleno desarrollo del hombre y su personalidad. Los segundos están determinados por la valoración que el sujeto hace de su propia vida. Se trabaja en la elaboración de una estrategia destinada a la modificación de los mitos y creencias erróneas generadas desde la aparición del VIH.

3. Un tercer conjunto de problemas que enfrentan este grupo de investigadores de la calidad de vida, es el de las dificultades inherentes a su evaluación. Su naturaleza bipolar objetiva-subjetiva y sus múltiples dimensiones, complejas e indeterminadas, otorgan a su evaluación diversos matices en función de la alternativa a ejecutar. Una primera propuesta incluirá el desarrollo de un instrumento específico para la evaluación de la calidad de vida en personas viviendo con VIH, que una vez validado, se utilizará en una estrategia de intervención para su mejoramiento.

\section{REFERENCI AS BI BLI OGRÁFICAS}

1. Hernández E. Calidad de vida y trasplante de órganos. En: Font Martinez M, editora. Atención psicológica en el trasplante de órganos. El trasplante de corazón. La Habana: Editorial Ciencias Médicas; 2008. p. 148-81.

2. González U. El concepto de calidad de vida y la evolución de los paradigmas de las ciencias de la salud. Rev Cubana de Salud Pública [Internet]. 2002 [citado1 Feb 2011];28(2): 6 . Disponible

en: http://scielo.sld.cu/scielo.php?script=sci arttext\&pid=S086434662002000200006\&lng=es\&nrm=iso\&ting=es

3. Dueñas D. Calidad de vida percibida en adultos mayores de la provincia Matanzas [tesis]. La Habana: Escuela Nacional de Salud Pública; 2007.

4. Miguel L. Evaluación de la Calidad de vida en personas infectadas por el Virus de Inmunodeficiencia Humana ( $\mathrm{VIH})$ [tesis]. Barcelona, Bellaterra: Facultad de Psicología, Universidad de Barcelona; 2001.

5. Canini SR, Marin da Reis, Bernardes dos Pereira R, Lucineia Alves G, Elucir Pela $\mathrm{N}$, Rotter T. Qualidade de vida de indivíduos com HIV/AIDS: uma revisão de literatura. Rer Latina-Amer Enfermagem. 2004; 12(6): 8.

6. Urzúa A. Calidad de vida relacionada con la salud: Elementos conceptuales. Rev Méd Chile [Internet]. 2011 Mar [citado 1 Feb 2011];138(3):358-65. Disponible en: http:// www.scielo.cl/scielo.php?script=sci_arttext\&pid=S0034-

98872010000300017\&lng=es doi: 10.4067/S0034-98872010000300017.

7. ONUSIDA 2010. Acción conjunta para obtener resultados [Internet]. Marco de resultados del ONUSIDA de 2009 a 2011 [citado 22 Sept 2011]. Disponible en: http://data.unaids.org/pub/BaseDocument/2010/jc1713_joint_action_es.pdf 
8. Pio de Almeida M, Fachel Leal O, Louzada S, Xavier M, Chachamovich E, Vieira G, et al. Desenvolvimento da versão em portugués do instrumento de avaliação de qualidade de vida da OMS (WHOQOL-100). Rev Bras Psiquiatr [Internet]. 1999 [citado 15 Sept 2001];21(1):11. Disponíveis à: http://www. scielo. br/scielo.php?script=sci arttext\&pid=S1516$\underline{44461999000100006}$

9. Hernández CL. Bioética Médica. Apuntes sobre calidad de vida. Hospital Universitario "Comandante Faustino Pérez". Matanzas: Centro Provincial de Información de Ciencias Médicas; 2003.

10. Moreno B, Ximénez C. Evaluación de la calidad de vida. En: Buela Casal G, editor. Manual de evaluación en Psicología Clínica y de la Salud. Madrid: Siglo XxI; 1996. p. 1045-70.

11. Guevara H, Dominguez A, Ortunio M, Padrón D, Cardozo R. Percepción de la Calidad de vida desde los principios de complejidad. Rev Cubana de Salud Pública [Internet]. 2010 [citado 1 Feb 2011];36(4):6. Disponible en: http://scielo.sld.cu/scielo.php?script=sci_arttext\&pid=S0864-

34662010000400011\&lng=es\&nrm=iso\&tlng=es

12. Bayarre $\mathrm{H}$. Las transiciones demográficas y la calidad de vida objetiva en la Tercera Edad. GEROINFO [Internet]. RNPS; 2010 [citado 25 Ene 2011];1(3): 29. Disponible

en:

http://www.sld.cu/galerias/pdf/sitios/gericuba/las_transiciones_demografica_y_epi demiologica_y la_calidad de vida_objetiva_en_la_tercera_edad.pdf

13. Gómez M, Sabeh Eliana N. Calidad de vida. Evolución del concepto y su influencia en la investigación y práctica [Internet]. 2006 [citado 20 Oct 2010]. Disponible en: http://campus. usal.es/ inico/investigacion /invesinico/calidad.htm

14. Hernández E. Calidad de vida y rehabilitación cardiovascular. En: Hernández Meléndrez $E$, editora. Intervención psicológica en las enfermedades cardiovasculares. Guadalajara: Universidad de Guadalajara; 2003.

15. Grau J A, Victoria CR, Hernández E. Calidad de vida y Psicología de la Salud. En: Hernández Meléndrez E, Grau Abalo J, editores. Psicología de la Salud. Fundamentos y aplicaciones. Guadalajara: Ediciones La Noche; 2005.

16. Malpica E, Ruesca Dominguez C. Reflexión sobre "Calidad de la Vida" en relación al "Valor de la Vida". Rev Médica Electrónica [Internet]. 2006 [citado 22 Nov 2010];28(5). Disponible en: http://www. revmatanzas.sld.cu/revista\% 20medica/ano\%202006/vol5\% 202006/te ma13.htm

17. ONUSIDA. Nuevo tratamiento evitaría millones de muertes. XVIII Conferencia internacional sobre el sida. Viena, 18 al 23 de Julio de 2010 [Internet]. 2010 [citado 22 Sept 2010]. Disponible en: http://www.enfoque365.net/N13728onusida-nuevo-tratamiento-millones-de 
18. Teva I, Bermúdez, María de la Paz, Hernández Quero, J; Buela Casal G. Calidad de vida relacionada con la salud en la infección por el VIH. Facultad de Psicología, Universidad de Granada, España, 2004. Disponible en: http://www.alapsa.org/revista/articulos/Gualberto_Buela-Casal.pdf Acceso en: 1 de Feb. 2011.

19. Gamba M. Intervención psicológica en PVIH y prestadores para modificar afrontamientos y actitudes. Hospitales Esperanza y Militar de Luanda. Angola, 2005-2009 [tesis]. La Habana: Escuela Nacional de Salud Pública; 2010.

Recibido 28 de abril de 2011.

Aprobado: 11 de octubre de 2011.

Lucas Antonio Nhamba. Calle García de Horta s/n, Edificio de las Finanzas. 5to. piso. Apto. E. Huambo, Angola.

Teléf.: +2442412 20342. Correo electrónico: nhambalu@yahoo.com.br, edelsys.hernandez@infomed.sld.cu 